\title{
Videoüberwachung in der Psychiatrie - Pro \& Kontra
}

\author{
Video Surveillance in Psychiatric Hospitals - Pro \& Contra
}

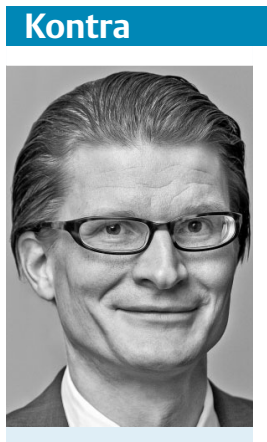

Klaus zu Hoene
Patienten in geschlossenen Abteilungen mit Videokameras zu überwachen, ist eine weitverbreitete Praxis in psychiatrischen Kliniken. Für eine persönliche Überwachung fehlt häufig das Personal. Doch diese Praxis verstößt gegen elementare Grundsätze des Datenschutzes, wegen des erheblichen Kontroll- und Einschüchterungspotenzials der Videoüberwachung. In der Psychiatrie darf sie deshalb nur in Einzelfällen angeordnet werden und ist in NordrheinWestfalen sogar gänzlich untersagt.

Nach $\S 4$ Abs. 1 Bundesdatenschutzgesetz (BDSG) ist die Erhebung, Verarbeitung und Nutzung personenbezogener Daten nur zulässig, soweit das Bundesdatenschutzgesetz oder eine andere Rechtsvorschrift dies erlaubt oder der Betroffene eingewilligt hat. Selbst wenn ein Patient einwilligungsfähig ist, wird es in den meisten Fällen an der Freiwilligkeit der Einwilligung fehlen. In Abhängigkeitsverhältnissen, etwa in einem Arbeitsverhältnis, halten Datenschützer eine freiwillige Einwilligung für nahezu ausgeschlossen. Wer zwangsweise aufgrund akuter Eigenoder Fremdgefährdung in die geschlossene Abteilung eines psychiatrischen Krankenhauses eingewiesen wird, kann schwerlich in eine Videoüberwachung freiwillig einwilligen. Aber auch bei Patienten, die sich freiwillig in eine geschlossene Behandlung begeben, kann von Freiwilligkeit der Einwilligung keine Rede sein, wenn etwa die Aufnahme in die Klinik von der Einwilligung abhängig gemacht wird.

Insofern bedarf die Videoüberwachung einer gesetzlichen Grundlage. Ausdrückliche Regelungen finden sich im Bundesdatenschutzgesetz oder den Datenschutzgesetzen der Bundesländer allerdings kaum. §6b BDSG erfasst nur die Überwachung öffentlich zugänglicher Räume wie etwa Bahnsteige, Ausstellungsräume eines Museums, Verkaufsräume oder Schalterhallen. Für Menschen mit psychischen Erkrankungen gelten in Deutschland spezielle Gesetze. So haben die einzelnen Bundesländer Gesetze über den „Schutz“ oder „Hilfen“ für psychisch kranke Menschen erlassen, zum Beispiel in Bayern das Unterbringungsgesetz und in Nordrhein-Westfalen das Gesetz über Hilfen und Schutzmaßnahmen bei psychischen Krankheiten (PsychKG). Nur dieses Gesetz enthält eine explizite Regelung zur Videoüberwachung, nämlich ein generelles Verbot [1].

2009 genehmigte der nordrhein-westfälische Sozialminister per Erlass die Videoüberwachung psychisch Kranker, um das Personal in den geschlossenen Abteilungen zu entlasten. Er hatte den Erlass auf $\S 6$ b BDSG gestützt, weil Klinikmitarbeiter die Zimmer der Patienten ohne deren Einverständnis betreten könnten, sodass es sich um öffentlichen Raum handele. Der Erlass löste eine politische Kontroverse und heftige Proteste von Betroffenenverbänden aus. Daraufhin wurde im November 2011 eine neue Bestimmung in §20 PsychKG-NRW aufgenommen (Abs.2): „Eine Beobachtung durch Einsatz technischer Mittel zur Anfertigung von Bildaufnahmen und Bildaufzeichnungen sowie zum Abhören und Aufzeichnen des gesprochenen Wortes ist verboten."

In den übrigen Bundesländern könnte man die Zulässigkeit aus den allgemeinen Vorschriften über die Anordnung besonderer Sicherungsmaßnahmen in den jeweiligen Unterbringungsgesetzen herleiten. Trotzdem verstößt die gängige Praxis vieler psychiatrischer Krankenhäuser, fixierte und isolierte Patienten regelmäßig mit Videokameras zu überwachen, gegen das Datenschutzrecht. Übergreifende Anforderungen an alle Maßnahmen, die den Datenschutz betreffen, sind nämlich deren Erforderlichkeit und Verhältnismäßigkeit. Es darf kein milderes Mittel zur Errei- chung des angestrebten Zwecks geben, welches das informationelle Selbstbestimmungsrecht weniger beeinträchtigt, und die Maßnahme darf nicht außer Verhältnis zu dem Zweck stehen.

Nach Auffassung medizinischer Experten ist die Videoüberwachung nicht nur ungeeignet, den angestrebten Zweck zu erreichen, sondern geradezu kontraproduktiv. Menschen, die stationärer psychiatrischer Behandlungen bedürfen, erleben schwere und akute Krisen und Verstörungen in Bezug auf die eigene Person und andere Menschen. Zu den häufigen Symptomen gehören Bedrohungsgefühle, wahnhafte Verkennungen, Misstrauen und paranoide Ängste. Die Betroffenen brauchen Rahmenbedingungen und persönliche Zuwendung, die ihnen helfen, sich sicherer zu fühlen und Vertrauen wiederzugewinnen. Eine Videoüberwachung verstärke jedoch das Gefühl von Schutzlosigkeit und Ausgeliefertsein. Über die medizinische Diskussion hat Morana Schütze eine Dissertation verfasst [2]. Sehr instruktiv sind auch die Protokolle der öffentlichen Sitzung des Ausschusses für Arbeit, Gesundheit, Soziales und Integration im nordrhein-westfälischen Landtag [3].

Als weniger einschneidende Maßnahme kommt nach Auffassung von Fachleuten vor allem die sog. Sitzwache in Betracht. Gerade in Ausnahmesituationen sei persönliche Betreuung nötig, um sich jederzeit ein Bild von der Verfassung des fixierten Patienten machen zu können. Betreuendes Personal müsse permanent als Ansprechpartner und zum schnellen Eingreifen bei einer Gefährdung, etwa durch beabsichtigte oder unbeabsichtigte Strangulation, zur Verfügung stehen. Bei isolierten Patienten ist ein Sichtfenster in das Krankenzimmer das mildere Mittel. Eine Überwachung rund um die Uhr ist auf diese Weise nicht zu gewährleisten, dürfte aber aus therapeutischen Gründen ohnehin nicht bei jedem Patienten angezeigt sein. 
Psychiatrische Kliniken sind auch nicht aus haftungsrechtlichen Gründen gehalten, Patienten in geschlossenen Abteilungen mit Videokameras zu überwachen, wie der folgende Fall belegt: Nachdem sich ein junger Mann mit einem Messer gefährliche Verletzungen zugefügt hatte, wurde er wegen des Verdachts auf psychotische Störungen in ein Intensivzimmer auf der geschlossen geführten Station eines Osnabrücker Klinikums aufgenommen. Er wurde mit Medikamenten behandelt und verhielt sich an den folgenden Tagen unauffällig. Bei einem Toilettengang verletzte er sich nach einem raptusartigen Impuls die Augen so schwer, dass er erblindete. Eine Klage des Patienten auf Schadensersatz wegen Verletzung von Aufsichtspflichten wurde vom Oberlandesgericht Oldenburg in letzter Instanz abgewiesen, weil bei Patienten mit dem geschilderten Befund während eines Toilettengangs nicht stets eine Begleitung oder Videoüberwachung erforderlich ist [4]. Das OLG macht sich in seiner Begründung folgende Ausführungen einer Sachverständigen zu eigen: „Derart einschneidende Maßnahmen wie eine Begleitung zu den Toilettengängen seien in Abwägung des dadurch zu erwartenden Nutzens für den Patienten nicht angezeigt gewesen. Eine Videoüberwachung erscheine gerade auf einer psychiatrischen Akutstation nicht sinnvoll.“

Nach alldem ist die Beobachtung mittels optisch-elektronischer Einrichtungen nur in Ausnahmefällen erlaubt, zur Abwehr spezifischer Gefahren für Leib und Leben der Patienten oder Dritten oder zur Verhinderung von Straftaten von erheblicher Bedeutung. Der verantwortliche Arzt muss im Einzelfall die Menschenwürde und das Recht auf informationelle Selbstbestimmung des Patienten gegen dessen Selbst- und Fremdgefährdungspotenzial abwägen. Gehen von einem isolierten Patienten erhebliche Gefahren aus, ist eine Videoüberwachung zu erwägen, wenn so vermieden werden kann, dass er fixiert werden muss; denn durch eine Fixierung würden dessen Freiheitsrechte wesentlich stärker eingeschränkt. Die gezielte Videoüberwachung bedarf der ärztlichen Anordnung. Anlass, Anordnung, Umfang und Dauer der Maßnahme sind zu dokumentieren und den Betroffenen oder deren gesetzlicher Vertretung mitzuteilen. Die Kameraaufnahmen sollten auf einem Monitor gezeigt werden, der nur einem begrenzten Personenkreis zugänglich ist, und dürfen nicht aufgezeichnet werden.

Legt der verantwortliche Arzt bei der Überwachung von Krankenzimmern durch Videokameras zu großzügige Maßstäbe an oder unterbleibt die einzelfallbezogene Abwägung ganz, weil die Überwachung pauschal angeordnet wird, drohen unter Umständen sogar strafrechtliche Konsequenzen. §201a StGB gilt nämlich unter anderem auch für ärztliche Behandlungszimmer und besonders gesicherte Hafträume in Justizvollzugsanstalten: „Wer von einer anderen Person, die sich in einer Wohnung oder einem gegen Einblick besonders geschützten Raum befindet, unbefugt Bildaufnahmen herstellt oder überträgt und dadurch deren höchstpersönlichen Lebensbereich verletzt, wird mit Freiheitsstrafe bis zu einem Jahr oder mit Geldstrafe bestraft.“

\section{Literatur}

1 In Thüringen ( $\$ 38$ ThürPsychKG) und Hamburg ( $\S 40$ a HmbMVollzG) bestehen Regelungen, die nur für den Maßregelvollzug gelten.

2 Schütze $M$. Untersuchung der Einstellung psychisch Erkrankter zur Videoüberwachung auf einer geschlossen geführten psychiatrischen Station. Dissertation. Bochum: 2011: http://www-brs.ub.ruhr-unibochum.de/netahtml/HSS/Diss/Schuetze Morana/diss.pdf

3 16. Sitzung des Ausschusses für Arbeit, Gesundheit, Soziales und Integration, vom 22. Juni 2011 (Landtag Nordrhein-Westfalen 4 - APr 15/236). http://www.juramagazin. de/In-den-Stellungnahmen-wurde-fernerargumentiert-dass-PersonalbetreuungKosten-verursache

4 Beschluss des OLG Oldenburg vom 17. Januar 2011 (Az. 5 U 187/10)

\section{Korrespondenzadresse}

\section{Dr. jur. Klaus zu Hoene}

Rechtsanwalt, Senior Consultant Datenschutz und

IT-Compliance, intersoft consulting services AG

khoene@intersoft-consulting.de

http://www.intersoft-consulting.de/

Bibliografie

Dol http://dx.doi.org/

10.1055/s-0032-1332940

Psychiat Prax 2013; 40: 118-119

(c) Georg Thieme Verlag KG

Stuttgart · New York

ISSN 0303-4259 